\title{
Risk groups in bladder cancer patients treated with radical cystectomy
}

\author{
Eva Mallen ${ }^{1}$, Pedro Gil2, Maria Jesus Gil2 \\ ${ }^{1}$ Department of Urology, Hospital Royo Villanova, Zaragoza, Spain; ${ }^{2}$ Deparment of Urology Hospital \\ Miguel Servet, Zaragoza, Spain
}

\section{ABSTRACT}

Objective: To stratify patients with bladder cancer into homogeneous risk groups according to statistically significant differences found in PFS (progression-free survival). To identify those patients at increased risk of progression and to provide oncological follow-up according to patient risk group.

Materials and Methods: A retrospective study of 563 patients treated with radical cystectomy (RC). In order to determine which factors might predict bladder tumour progression and death, uni- and multivariate analyses were performed. The risk groups were identified according to "inter-category" differences found in PFS and lack of differences, thus revealing intra-category homogeneity.

Results: Median follow up time was 37.8 months. Recurrence occurred in a total of 219 patients $(38,9 \%)$. In $63 \%$ of cases this was distant recurrence.

Only two variables retained independent prognostic value in the multivariate analysis for PFS: pathological organ confinement and lymph node involvement. By combining these two variables, we created a new "risk group" variable. In this second model it was found that the new variable behaved as an independent predictor associated with PFS. Four risk groups were identified: very low, low, intermediate and high risk:

- Very low risk: pT0 No

- Low risk: pTa, pTis, pT1, pT2 and pNo

- Intermediate risk: pT3 and pNo

- High risk: pT4 N0 or pN1-3.

Conclusions: We retrospectively identified 4 risk groups with an independent prognostic value for progression-free survival following RC.

Differences in recurrence patterns after RC between risk groups have led us to set different intervals in monitoring for cancer.

\section{ARTICLE INFO}

\section{Key words:}

Urinary Bladder Neoplasms;

Cystectomy; Pathology

Int Braz J Urol. 2015; 41: 30-9

Submitted for publication:

January 26, 2014

Accepted after revision:

February 02, 2014

\section{INTRODUCTION}

Bladder cancer is major health problem in Spain, with high incidence and elevated mortality (1-3). Radical cystectomy is the standard treatment for patients with muscle-invasive bladder cancer, however this is generally insufficient. In fact, it is very important to identify those at high risk of progression because these patients could benefit from adjuvant treatment and closer monitoring. Other authors $(4,5)$ have opted to divide patients into homogeneous risk groups according to statistically significant differences found in PFS and CSS (cancer-specific survival). A novel development in this study is the identification of the new risk group "Very low risk", which includes patients with a lower probability of suffering 
from disease progression. These patients are therefore less likely to require adjuvant treatment, and follow-up intervals may also be more distanced. We propose a follow-up strategy for RC-treated bladder cancer patients that identifies most cases of recurrence while at the same time avoids monitoring patients too closely. This implies in a reduction of costs related to tests and number of visits.

While it is true that our study is not a multidisciplinary project, we believe that our single-center study includes a sample sufficiently large to draw conclusions similar to those drawn from studies undertaken by multidisciplinary groups (6). The aim of the present study was establish risk groups and tailor follow-up accordingly with a schedule appropriate to the likelihood of progression.

\section{MATERIALS AND METHODS}

\section{Patient Population}

We retrospectively reviewed all patients who underwent radical cystoprostatectomy and pelvic lymphadenectomy for bladder cancer with curative intent at Miguel Servet University Hospital between 1975 and 2007. The study population consisted of 599 patients who underwent radical cystectomy. Thirty-six patients were eliminated from the study due to missing data. Therefore, analysis was performed on the 563 remaining patients.

Radical cystoprostatectomy and lymphadenectomy were always performed according to standard protocol; indications for cystectomy did not change during the time period studied. Cystectomy is indicated in patients with invasive bladder carcinoma, endoscopically uncontrollable superficial bladder cancer and high risk bladder tumors and for those with BCG-resistance bladder tumors. Radical cystectomy and limited pelvic lymph node dissection were performed in 84\% of the patients. In only 10 cases it was performed an extended pelvic lymphadenectomy. It is true that currently, this procedure is routinely performed in most cases.

All cystectomy specimens were subjected to routine pathological examination. In the last two decades the same pathologist examined the specimens microscopically. Primary tumors and lymphadenectomy were restaged based on the 2002 UICC TNM system.

\section{Statistical analysis}

"FileMaker Pro 11.0, version 11.0 v2" (FileMaker Inc) was used as database software and "PASW Statistics 18, version 18.0.0" (IBM ${ }^{\circ}$ ) was used as statistics software.

Paper-based patient records were reviewed and data were analyzed for possible predictive factors. Univariante analysis was performed using the Kaplan-Meier (or Mantel-Haenszel) test. Significant variables $(\mathrm{p}<0.05)$ and those close to significance $(\mathrm{p}<0.1)$ from univariate analysis were analyzed using backwards multivariate analysis with the Cox proportional hazards regression model. To search for a clinical application and to check the strength of the model, a new model was set up, with the combination of the statistically most powerful variable taken from multivariate analysis. We created a new variable called "risk groups", to try to identify any differences in PFS and CSS between the different categories of this variable. The risk groups identified were compared using the Kaplan-Meier method and log-rank test.

\section{RESULTS}

Cystectomy was indicated for muscle-invasive bladder cancer (MIBC) in 493 patients. In the remaining 70 patients, the indication for cystectomy was superficial bladder cancer. In these 70 cases, 33 underwent cystectomy for multifocal recurrence of NMBC (non muscle-invasive bladder cancer) such as TaG1-2 T1G1-2, 36 patients for recurrent high-grade tumours (TaG3, T1G3, and CIS) and in one case the reason for cystectomy was not specified. These data are summarized in the chart below (Figure-1).

The variables investigated in patients treated with cystectomy in the period of study were grouped into: pre-, peri- and post-cystectomy variables, as shown in Table-1.

At the time of cystectomy, median patient age was 65.3 years (IQR 13.1). In the analysis by decades it can be seen that patients were progressively older, with statistically significant differences.

Median follow-up time was 37.8 months (IQR 83.4, range 1,1-288,2). Of note are those variables that underwent changes over the decades 
Figure 1 - Indication of radical cystectomy.

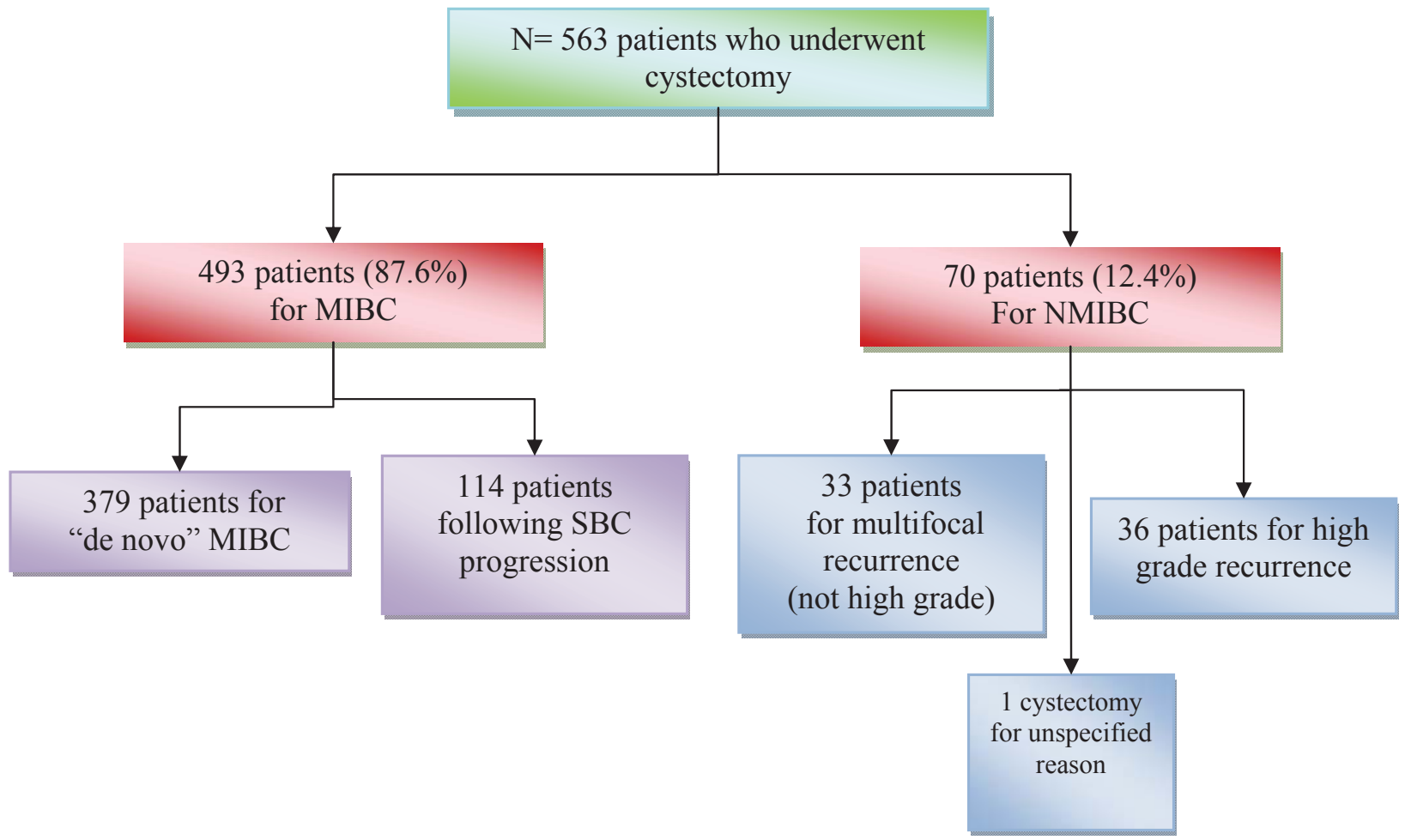

Table 1 - Variables studied.

\begin{tabular}{lcc}
\hline Variables Pre-Cystectomy & Variables Peri-Cystectomy & Variables Post-Cystectomy \\
\hline Gender & Transfusion & Hospital stay \\
Age & Type of catheterisation & Follow-up \\
Smoker & Ureteral reimplantation & Adjuvant CT \\
Alcohol & Pathological stage (pT) & Major perioperative complications \\
Risk occupation & Tumour grade & Minor perioperative complications \\
Living environment & Lymph node involvement (pN) & Late-onset complications \\
Comorbidity & Presence of ClS & Tumour recurrence in UUT \\
Clinical presentation & p53 & \\
Clinical stage of TURBT & Anatomical pathology Terminal ureter & \\
History of UC in UUT & Ureterectomy & \\
Neoadjuvant CT & Anatomical pathology type & \\
& Organ confinement & \\
& Tumour in UUT concomitantly with BC & \\
\hline
\end{tabular}

TURBT = Transurethral resection of bladder tumour; $\mathbf{S B C}=$ superficial bladder cancer; $\mathbf{U C}=$ urothelial carcinoma; UUT = upper urinary tract; CT: chemotherapy; $\mathbf{B C}=$ Bladder cancer 
studied, such as for example the number of units transfused $(\mathrm{p}<0,00)$, length of hospital stays and the number of complications $(p<0.03)$. All these variables decreased in number/duration. Conversely, continent urinary derivations $(\mathrm{p}<0.00)$ increased during the study period. In terms of studied tumor characteristics no clear differences were observed over the four decades of the study. In the percentage of patients with organ-confined tumors there were no statistically significant differences $(p=0.714)$. In the case of lymph node involvement prevalence increased over the decades, but not significantly $(\mathrm{p}=0.250)$.

Progressive disease occurred in 219 patients of the total series (38.9\%). We classified recurrence as: local, regional lymph node and distant. Local recurrence: we considered this to be the appearance of local recurrence in the pelvis urothelial tumor, surgical site and/or urinary tract without distant involvement. Locoregional nodal recurrence: this included pathological lymph node involvement and distant metastasis.

In patients who progressed, the predominant type of progression was distant metastasis in 63\% of cases. Mean PFS (progression-free survival) in the 563 patients was 157 (144.2-169.9) months (the median could not be calculated).

It can be observed that almost all events (recurrence from bladder cancer) occurred in the first two years of follow up. In fact, by the end of the second year of follow-up, 77\% of the events had already occurred. There was a 73\% probability of survival in the first year, falling to 55\% at the end of the fifth year, with little further change to the end of the tenth year (51\%) (Figure-2 and Table-2).

In patients who progressed, $\mathrm{n}=219$ (38.9\%), median progression-free survival was 9.7 months (CI 95\% 8.3-11.1); there were no differences in PFS by type of progression. Progression appeared to occur shortly before lymph node recurrence (median PFS=8.6 months [6.3-11]), but significant differences were found in the univariate analysis.

Univariante analysis demonstrated that pathological organ confinement, lymph node involvement, tumour grade, terminal ureter involvement and the administration of adjuvant chemotherapy were variables that were significantly
Figure 2 - PFS in 563 patients.

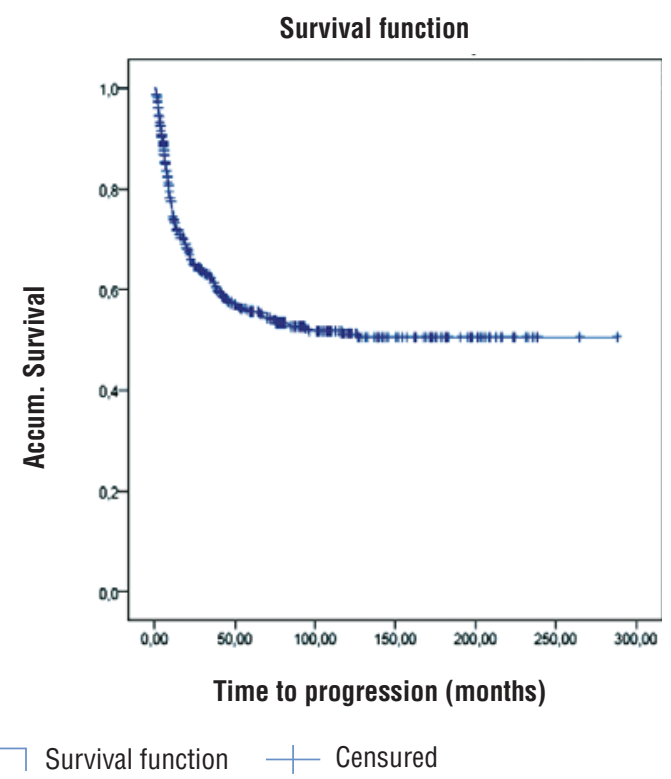

Table 2 - Mean PFS in the whole series.

\begin{tabular}{lc}
\hline$T$ (months) & $\begin{array}{c}\text { Cumulative survival } \\
\text { probability }\end{array}$ \\
\hline $12 \mathrm{~m}$ & $83.1 \%$ \\
$36 \mathrm{~m}$ & $65.5 \%$ \\
$60 \mathrm{~m}$ & $61.5 \%$ \\
\hline $120 \mathrm{~m}$ & $55.2 \%$ \\
\hline
\end{tabular}

associated with lower progression-free survival (Table-3).

In the multivariate analysis (Table-4) we entered the significant variables detected in the univariate analysis and found that pathological organ confinement and lymph node involvement were independent variables that were significantly associated with PFS.

In order to identify patients at increased risk of urothelial disease progression or recurrence following cystectomy, we developed a classification system based on the multivariate predictive variables for PFS: pathological organ confinement and lymph node involvement. We created a new variable called "risk groups", using a combination of the two previous variables ( $\mathrm{pT}$ and $\mathrm{pN}$ ), in order 
Table 3 - Univariante analysis of predictive factors for PFS.

\begin{tabular}{|c|c|c|c|c|c|}
\hline Long rank (Mantel-Cox) & $\mathrm{Chi}^{2}$ & Df & Sig. & Variable & $\%$ patients \\
\hline \multirow[t]{2}{*}{ Organ confinement } & 66.035 & 1 & 0.000 & $\leq \mathrm{pT} 2$ & $54 \%$ \\
\hline & & & & $>$ pT2 & $46 \%$ \\
\hline \multirow[t]{2}{*}{ Pathological lymph node status } & 105.106 & 1 & 0.000 & pNO & $74 \%$ \\
\hline & & & & $\mathrm{pN}+$ & $26 \%$ \\
\hline \multirow[t]{2}{*}{ Tumour grade } & 8.651 & 2 & 0.013 & $G_{1}-G_{2}$ & $27 \%$ \\
\hline & & & & G3 & $73 \%$ \\
\hline \multirow[t]{2}{*}{ Terminal ureter } & 5.621 & 1 & 0.018 & Normal & $90 \%$ \\
\hline & & & & pathological & $10 \%$ \\
\hline Adjuvant CT & 7.511 & 1 & 0.006 & - & $6 \%$ \\
\hline
\end{tabular}

PFS = progression-free survival; $\mathbf{C T}$ = chemotherapy

Table 4 - Multivariate analysis. Predictive model for PFS.

\begin{tabular}{|c|c|c|c|c|c|c|c|c|}
\hline \multirow[t]{2}{*}{ Variable } & \multirow[t]{2}{*}{ B } & \multirow[t]{2}{*}{ SE } & \multirow[t]{2}{*}{ Wald } & \multirow[t]{2}{*}{$\mathrm{Gl}$} & \multirow[t]{2}{*}{$p$} & \multirow[t]{2}{*}{ O.R. } & \multicolumn{2}{|c|}{$95 \% \mathrm{Cl}$ for $\operatorname{EXP}(\mathrm{B})$} \\
\hline & & & & & & & Lower & Upper \\
\hline Pathological organ confinement & 1.077 & 0.283 & 14.445 & 1 & 0.000 & 2.936 & 1.685 & 5.117 \\
\hline Lymph node involvement & 0.861 & 0.214 & 16.219 & 1 & 0.000 & 2.365 & 1.556 & 3.595 \\
\hline
\end{tabular}

PFS $=$ progression-free survival

to identify any differences in PFS between the different categories of this variable.

We analysed the survival for each pT variables (pT0 vs. pTa-Tis-T1 vs. pT2 vs. pT3-4) and $\mathrm{pN}$ variables (pNo vs pN1-3). We observed clear differences between the patients with pT0 and the group stages PTa, pTis, pT1, pT2, and of course among the rest of groups, which led us to differentiate between a group of very low risk, separating it from the rest. According to this analysis, four independent groups were identified (Table-5).

With respect to the survival graph $(\mathrm{Fi}-$ gure-3), firstly it can be seen that there are statistically significant inter-category differences $(p<0.001)$, which appear to confirm the homogenous nature of the composition of the four categories. Of note are the findings in PFS in the
Table 5 - Risk groups.

\begin{tabular}{lc}
\hline "Risk group" variable & \\
\hline Categories & Description \\
\hline Very low risk & pT0 and pNO \\
Low risk & pTa, pT1, pTis or pT2, and pNO \\
Intermediate risk & pT3 and pNO \\
High risk & pT4N0 or pN1-3 \\
\hline
\end{tabular}

"Intermediate risk" and "High risk" groups. It is particularly striking that events occur so early in these groups. After two years of follow-up, 84\% (45 out of 53) events relating to progression have appeared in the "Intermediate risk" group, and $84 \%$ (89 out of 105) in the "High risk" group. 
Figure 3 - PFS by risk group.

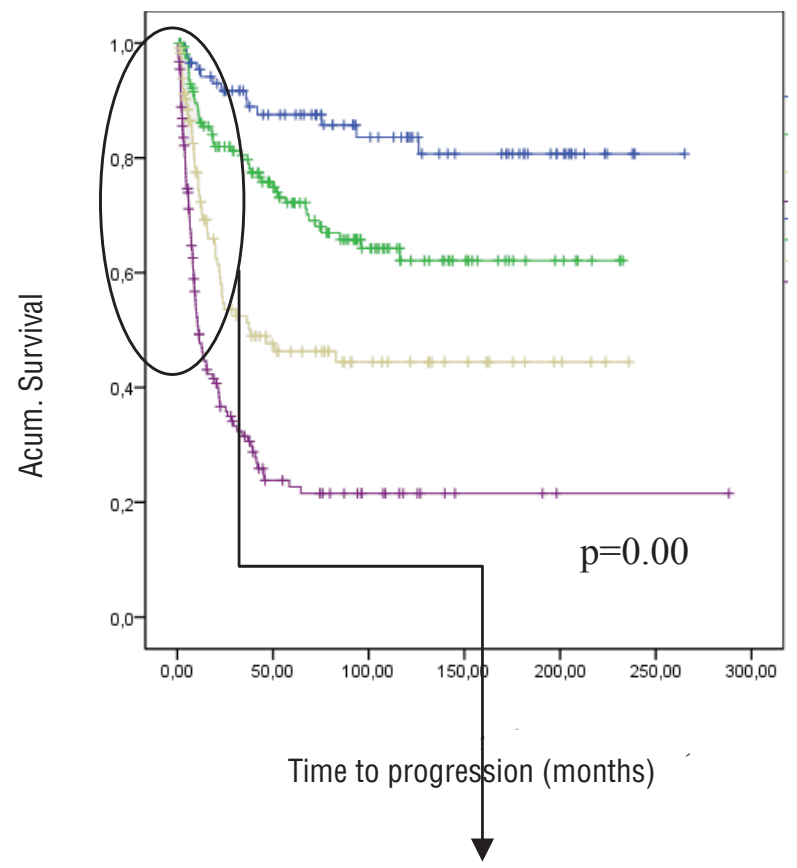

Risk group

Very low risk

Low risk

Intermediate risk

High risk

Survival function

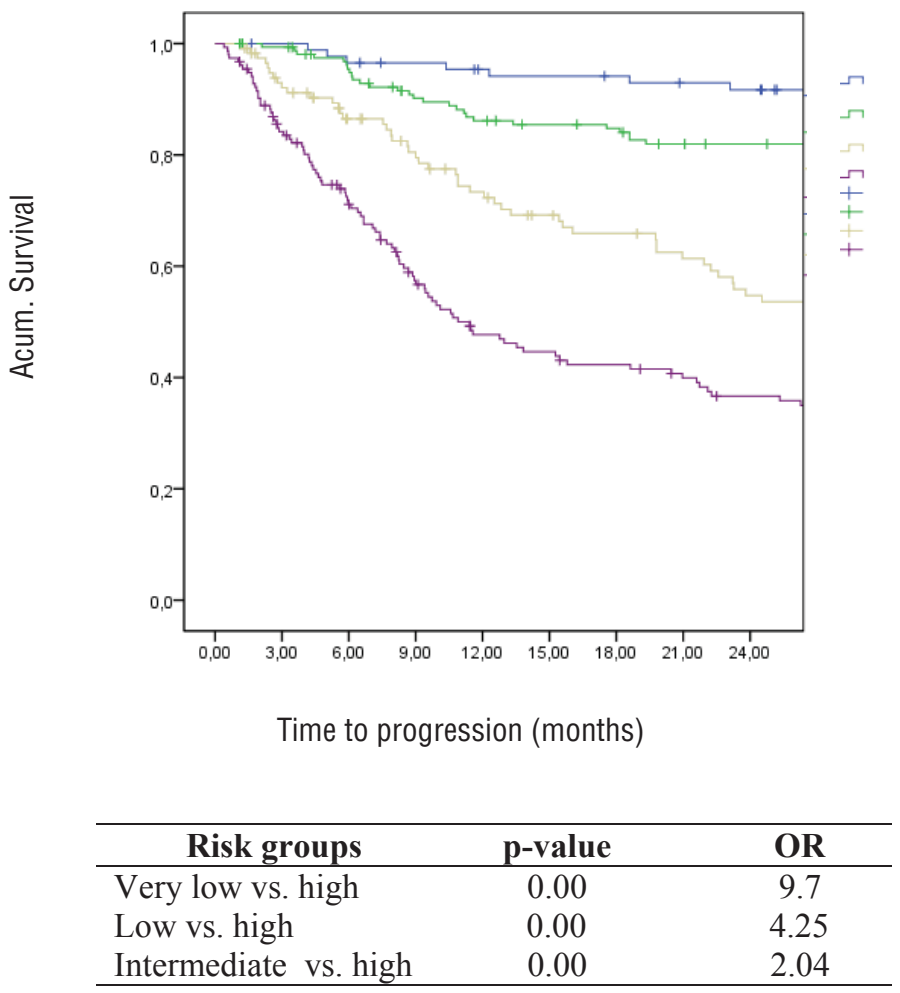

Risk group

Very low risk

Low risk

Intermediate risk

High risk 
The survival graphs show that the risk of progression stabilizes. This is similarly reflected in the survival table (Table-6). This shows the probability of reaching a certain time point, in the post-cystectomy follow-up, without progression.

In this second model it was found that the new "risk group" variable behaved as an independent predictor associated with PFS.

From the data of Table-7 it can be deduced that a patient in the high risk group has an OR of progression that is 9.7 times $(1 / 0.103)$ higher than patients with a very low risk. The same high risk patient has an OR that is 4.25 times $(1 / 0.235)$ higher for progression than low-risk patients.

\section{DISCUSSION}

This study was undertaken primarily due to the high rates of incidence and mortality of bladder cancer in Spain and in our region, Aragon. Rates are above the mean for Europe $(7,8)$, and this, together with the marked ageing of population, suggests that the upward trend will continue.
In the literature there are several studies on the prognostic value of different variables such as stage, lymph node and lymphovascular involvement, type of urinary diversion, margin status, etc. on invasive bladder cancer progression and survival. However, organ confinement and lymph node involvement are repeatedly found to be independent risk factors for PFS and cancer-specific mortality in most series $(4,9,10)$.

In a study at Gregorio Marañón Hospital (4), both pathological stage and lymph node involvement were found to be independent predictors for cancer-specific survival. In that study the authors, like us, also constructed a second model by grouping local stage (pT) and lymph node involvement $(\mathrm{pN})$ into risk groups in order to study CSS. Thus, the association by risk group allowed them to predict the risk of death from bladder cancer more reliably, and to identify patients in whom cystectomy is insufficient and who could benefit from adjuvant treatment.

Solsona (5) also stratified patients by risk. In this study, the records of 298 patients who un-

Table 6 - Survival table (PFS) by "Risk group".

\begin{tabular}{llcccccc}
\hline \multicolumn{7}{c}{ PFS by “Risk group”. Survival table } \\
Categories & & $\begin{array}{c}\text { End } \\
\text { 1st year }\end{array}$ & $\begin{array}{c}\text { End } \\
\text { 2nd year }\end{array}$ & $\begin{array}{c}\text { End } \\
\text { 3rd year }\end{array}$ & $\begin{array}{c}\text { End } \\
\text { 4th year }\end{array}$ & $\begin{array}{c}\text { End } \\
\text { 5th year }\end{array}$ & $\begin{array}{c}\text { End } \\
\text { 10th year }\end{array}$ \\
\hline Very low & pT0 and pN0 & 0.954 & 0.917 & 0.903 & 0.875 & 0.875 & 0.836 \\
Low & pTis, pTa-1, pT2 and pN0 & 0.861 & 0.82 & 0.797 & 0.758 & 0.722 & 0.621 \\
Intermediate & pT3 and pN0 & 0.734 & 0.547 & 0.525 & 0.476 & 0.463 & 0.444 \\
High & pT4 or pN1-3 & 0.485 & 0.367 & 0.315 & 0.238 & 0.227 & 0.215 \\
\hline
\end{tabular}

Table 7 - Subvariables that retain independent statistical significance for PFS.

\begin{tabular}{lcccccccccc}
\hline Variable & & B & ET & Wald & gl & P & Exp (B) & \multicolumn{2}{c}{$95 \%$ Cl forEXP(B) } \\
\cline { 8 - 11 } & & & & & & & & Lower & Upper \\
\hline Risk Group & High vs very low & 2.27 & 0.296 & 59.088 & 1 & 0.00 & 0.103 & 0.58 & 0.184 \\
& $\quad$ High vs low & 1.44 & 0.177 & 66.600 & 1 & 0.00 & 0.235 & 0.166 & 0.333 \\
& High vs intermediate & 0.715 & 0.169 & 17.853 & 1 & 0.00 & 0.489 & 0.351 & 0.682 \\
\hline
\end{tabular}


derwent cystectomy were retrospectively analysed and risk groups were established based on lymph node involvement, pathological stage and prostatic stromal involvement as predictors of mortality in the multivariate analysis. Their figures for 5-year CSS, by risk group, were $86.4 \%$ for the low risk group (P1-2NOSt-), 64.4\% for the intermediate risk group (60.9\%-65.3\%) (P1-2N1St-, P3N0St-, $\mathrm{HR}=2.7)$ and $28.1 \%(0 \%-47.7 \%)$ for the high risk group (N2-3, P4, St+, N1P3, HR=8.7). These figures were not far off our 5-year CSS of 89\% for the very low risk group, 75\% for low risk, 54\% for intermediate and 30\% for low grade (data not shown).

Sonpavde (11) investigated bladder cancer risk following cystectomy using pathological factors to facilitate an indication for adjuvant treatment. This series was more similar to ours in terms of sample size and study time (although the follow-up period was longer in their study), and it was also found that pathological stage was a predictor. Like us, these authors constructed a model with prognostic groups and found that stage, lymphovascular involvement and poorly-differentiated cells were predictors of PFS. They distinguished three groups according to the presence of the three variables in the multivariate analysis, assigning a score of $0-4$, according to the presence of these variables, and depending on the cumulative score, they divided patients into low, intermediate and high risk groups.

In the case of the Abol-Enein and Ghoneim group (12), which involved a large series because of the high incidence of bladder cancer in Egypt, these authors concluded that PFS predictors are lymph node involvement, stage, lymphovascular invasion and type of urinary diversion. Using these four variables, patients were stratified into four risk groups ranging from low risk (T1,N0, LV-, orthotopic diversion) to maximum risk ( $44, \mathrm{~N}+, \mathrm{LV}+$, rectal diversion), comparing survival curves and likelihood of progression among these groups. They found a 5-year PFS of $64.5 \%$, which does not differ greatly from our figure of 55.6\%. Like these authors, we divided patients into categories or risk groups, with two objectives. Firstly, in reference to follow-up we aimed to identify risk of progression and detect this as early as possible. The second objective was to identify candidates for adjuvant treatment, with the final objective of increasing CSS.

In our study, differences in progression patterns after radical cystectomy suggest the need for varied follow-up protocols for each group. We proposed a stage-based protocol for monitoring of patients with bladder cancer treated with radical surgery that captures most recurrences while limiting over-investigation. Multicenter studies are consistent with our study, showing that in most patients the tumor recurs in the first two years after radical cystectomy and over half of these recurrences are distant (6).

Table- 6 shows the probabilities of progression-free survival at a certain time point according to risk group. Looking at how to apply these data in clinical practice, we can draw up guidelines and recommendations for monitoring these patients. To date, there has been no consensus on how to follow up these patients after surgical intervention. Available guidelines include the National Comprehensive Cancer Network and the European Association of Urology recommendations, which acknowledge the need for risk-stratified surveillance but do not clearly delineate any protocols $(13,14)$.The ESMO (European Society for Medical Oncology) guidelines, by contrast, do not address any stage-specific surveillance regimen (15).

In the very low risk group, patients could be followed up less frequently from the second year onwards. This would not be recommendable in the low risk group until the third year, when the probability of progression becomes stable. In contrast, patients in the intermediate and high risk groups should continue six monthly follow-ups until the fourth or fifth year. However, there is a high probability of progression and early-onset of events in the intermediate and high risk groups during the first months of follow-up, especially in the high risk group. 15\% of patients in this group experience progression by the end of the third month and $48 \%$ by the end of the first year. The risk of progression is almost 10 times higher than in the very low risk group and two times higher in comparison with the intermediate risk group. For this reason we suggest three-monthly follow-up for the first year in the high risk group, as shown in Table-8. Later, the probability of progression drops, 
Table 8 - Follow-up recommendations.

\begin{tabular}{lcccccc}
\hline Risk group & 1st year & 2nd year & 3rd year & 4th year & 5th year & 6th-10th year \\
\hline Very low & Six monthly & Six monthly & Yearly & Yearly & Yearly & Yearly \\
Low & Six monthly & Six monthly & Six monthly & Yearly & Yearly & Yearly \\
Intermediate & Four monthly & Six monthly & Six monthly & Six monthly & Yearly & Yearly \\
High & Three monthly & Six monthly & Six monthly & Six monthly & Yearly & Yearly \\
\hline
\end{tabular}

so follow-up can be performed at six-monthly intervals until the probability of progression stabilises (in the 4th year). In the low risk group, patients could be followed up less frequently from the third year onwards, when the probability of progression stabilises. Faysal et al. (6) proposed stage-based protocols for surveillance of patients with bladder cancer based on recurrence patterns that coincide with our group in terms of the frequency of visits especially in the first year of follow-up in patients with very high risk of recurrence.

Since there is such a high probability of progression in the intermediate and high risk groups, intensive follow-up of these patients is clearly essential. However, the aim must be to prevent progression from occurring or to delay it as much as possible. Therefore, an assessment should be made of whether to administer adjuvant chemotherapy in these two groups of patients.

It is true that there are no randomised trials that demonstrate the superiority of adjuvant chemotherapy in MIBC treatment, but until such studies are available, we would recommend that chemotherapy - preferably within the framework of a clinical trial - as it is one of the few tools at our disposal that lengthens CSS.

Our study has several potential limitations, including those inherent to any retrospective study. For example, the variable margin status; this was not studied in the early years of the study period and therefore could not be included in the study. Extent of surgery, such as the upper limit of lymph node dissection, changed slightly and was not consistent during the study period. Our median follow-up time may seem short (37.8 months) however these are patients with an ominous prognosis. Further- more, this is a single institution study. Findings must be evaluated externally in a larger patient cohort and validated prospectively for practical use.

\section{CONCLUSIONS}

Non-organ confinement and lymph node involvement in radical cystectomy specimens are factors that retain independent prognostic value in progression-free survival in the multivariate analysis. We retrospectively identified four risk groups (very low, low, intermediate and high) with an independent prognostic value for progression-free survival following radical cystectomy. This would be useful in order to provide information to patients and physicians and to improve stratification for future clinical trials. This would serve to optimize indications for treatment, avoiding excessive monitoring and reducing costs.

\section{ABBREVIATIONS}

PFS $=$ progression-free survival

$\mathrm{RC}=$ radical cystectomy

CSS $=$ cancer-specific survival

MIBC $=$ muscle-invasive bladder cancer

$\mathrm{NMBC}=$ non muscle-invasive bladder cancer

\section{CONFLICT OF INTEREST}

None declared.

\section{REFERENCES}

1. [No Authors] Asociacion Spanish Cancer. Available at: http:// www.todocancer.com 
2. National Epidemiology Center. Area Environmental Epidemiology and Cancer. Cancer mortality in Spain. Consulted 2011. Available at: http://cne.isciii.es

3. World Health Organization. Available at: http://www.who.int

4. Monzó Gardiner JI, Herranz Amo F, Díez Cordero JM, Cabello Benavente R, Silmi Moyano A, Hernández Fernández C. Prognostic factors for survival in patients with transitional bladder cancer treated with radical cystectomy. Actas Urol Esp. 2009;33:249-57.

5. Solsona E, Iborra I, Dumont R, Rubio J, Casanova JL, Almenar S. Risk groups in patients with bladder cancer treated with radical cystectomy: statistical and clinical model improving homogeneity. J Urol. 2005;174:1226-30.

6. Yafi FA, Aprikian AG, Fradet Y, Chin JL, Izawa J, Rendon R, et al. Surveillance guidelines based on recurrence patterns after radical cystectomy for bladder cancer: the Canadian Bladder Cancer Network experience. BJUI. 2012;110:13171324.

7. Serrano P. Study of bladder cancer in the health area II of the province of Zaragoza: Epidemiology, anatomo and clinical outcome prediction model for the period 19942003 [MS thesis]. University of Zaragoza, 2007.

8. Romero FJ, Bernal M. Evolución en el carcinoma vesical en el área III de Zaragoza en los últimos 30 años. Póster XXVII Reunión del Grupo de Urología Oncológica, Sevilla, 2010.
9. Bassi P, Ferrante GD, Piazza N, Spinadin R, Carando R, Pappagallo $\mathrm{G}$, et al. Prognostic factors of outcome after radical cystectomy for bladder cancer: a retrospective study of a homogeneous patient cohort. J Urol. 1999;161:1494-7.

10. Frazier HA, Robertson JE, Dodge RK, Paulson DF. The value of pathologic factors in predicting cancer-specific survival among patients treated with radical cystectomy for transitional cell carcinoma of the bladder and prostate. Cancer. 1993;71:39934001.

11. Sonpavde G, Khan MM, Svatek RS, Lee R, Novara G, Tilki $D$, et al. Prognostic risk stratification of pathological stage T2NO bladder cancer after radical cystectomy. BJU Int. 2011;108:687-92.

12. Baco E, Rud E, Vlatkovic L, Svindland A, Eggesbø HB, Hung $A J$, et al. Predictive value of magnetic resonance imaging determined tumor contact length for extracapsular extension of prostate cancer. J Urol. 2015;193:466-72.

13. Stenzl A, Witjes JA, Comperat E, et al.Guidelines on Bladder Cancer 2012: Muscle-invasive and Metastatic. Available at http://www.uroweb.org. Accessed November 2012.

14. NCCN Clinical Practice Guidelines in Oncology (NCCN Guidelines TM). Bladder Cancer. 2012. Available at: http:// www.nccn.com. Accessed November 2012.

15. Bellmunt J, Albiol S, Kataja V; ESMO Guidelines Working Group. Invasive bladder cancer: ESMO clinical recommendations for diagnosis, treatment and follow-up. Ann Oncol. 2008;19 Suppl 2:ii47-8.

\footnotetext{
Correspondence address: Eva Mallen, MD Department of urology Hospital Royo Villanova Avenida San Gregorio, 30, Zaragoza, 50015, Spain E-mail: evamallen@hotmail.com
} 